\title{
An overview about elbow instability
}

\author{
Roberto Rotini
}

Published online: 23 March 2014

(C) Istituto Ortopedico Rizzoli 2014

Promoting and encouraging in a growing number of members of our Society the passion for elbow surgery is a precise dictate of the SICSeG Board and one of my heartfelt tasks during these 2 years as president. We feel the precise need to fill the cultural gap developing in the recent years between the "so much investigated shoulder" and the "too often neglected elbow."

One of the current and most debated topics in elbow surgery is certainly instability.

In the recent years, considerable improvements have come in the biomechanical knowledge about the role of elbow stabilizers. The complex interactions among the different stabilizers when injured at the same time have been better understood.

Anyway, uncertainties about both nomenclature and classification still exist in the definition of the different patterns of injury.

Even apparently simple concepts like instability and dislocation can be in some clinical settings so intricate as to make it difficult to give a separate and specific definition of each of them. We can describe dislocation/subluxation as a static condition of joint incongruency, generally produced by a lesion of a number of stabilizers, and instability as the dynamic condition in which-due to the lesion of one or more stabilizers-a reduced joint can loose its congruency (dislocation/subluxation).

An elbow can be "reduced-stable" (normal condition), "reduced-unstable" (e.g., acute simple dislocation when the reduced elbow is immobilized in $90^{\circ}$ flexion with hand in pronation), "non-reduced-unstable" (e.g., terrible triad), or "non-reduced-stable" (persistent chronic dislocation).

R. Rotini $(\bowtie)$

Istituto Ortopedico Rizzoli, Bologna, Italy

e-mail: roberto.rotini@ior.it
In these years classification systems have been proposed which separate the different patterns of lesion based on the injured stabilizers $[1,2]$.

It is my opinion that among the different classifications the one presented during the 2013 Rizzoli Elbow Course and approved by Prof. Shawn O'Driscoll (Table 1) is at the present time the most practical and complete one in order to clearly and simply define the main patterns of dislocation and instability. This classification separates the concepts of dislocation and of instability and takes into account three main features:

- lesion of the ligaments only (simple instability) or fracture-dislocation (complex instability).

- direction and mechanism of instability/dislocation.

- timing: acute, chronic, and recurrent dislocation.

Simple elbow instability indicates a dislocation with soft tissue lesions without associated fractures that can compromise joint stability [3, 4]. In the great majority of cases simple instability is a posterior dislocation produced by a posterolateral rotation mechanism (PLRI) as described by O'Driscoll [5]. Cases of anterior or divergent dislocation are on the contrary very rare. There is nowadays wide agreement that the treatment of this condition should be conservative, except for the rare cases where reducing the elbow or holding its reduction proves not possible.

The term Complex elbow instability, which replaces the former "fracture-dislocation" and "transolecranon fracture," means on the other side the association of ligaments and bony lesions. As a general rule, when the bony damage is bigger, the ligaments lesions are more contained and vice versa. This helps to explain situations where minor bone fractures, e.g., of the coronoid, are associated with severe elbow instability. Also most of the complex elbow instabilities take place with a posterolateral rotatory 
Table 1 Elbow dislocation and instability classification

\begin{tabular}{llll}
\hline Elbow dislocation & & & \\
\hline $\begin{array}{l}\text { With or without } \\
\text { fracture }\end{array}$ & $\begin{array}{c}\text { Simple } \\
\text { instability }\end{array}$ & $\begin{array}{c}\text { Complex } \\
\text { instability }\end{array}$ & \\
$\begin{array}{l}\text { Direction and } \\
\text { mechanism of } \\
\text { dislocation }\end{array}$ & $\begin{array}{c}\text { Posterior: } \\
\text { Anterior }\end{array}$ & Divergent \\
& - PLRI & & \\
& - PMRI & & \\
Timing & - Direct & & \\
& posterior & & \\
& Acute: & Chronic: & \\
& - Reducible & - Neglected & \\
& stable & & \\
& - Reducible & - Not adequately \\
treated
\end{tabular}

mechanism: the most frequent patterns are characterized by joint dislocation/subluxation, lateral collateral ligament (LCL) lesion, and fracture of the radial head with or without associated coronoid fracture (terrible triad). The cases of complex elbow instability caused by a posteromedial rotatory mechanism are rare, and they take place with elbow subluxation, injury of the anteromedial coronoid facet, and lesion of the lateral ligament complex [6]. Also fracture-dislocations, characterized by olecranon fracture that usually are due to a direct injury to the joint, are rather rare.

Chronic or persistent elbow dislocations can be very rarely caused by the absence of initial treatment (neglected conditions, unrecognized, or untreated) or more frequently can originate from an inadequate treatment for the elbow stabilizers (usually the coronoid, the radial head, and the LCL). Persistent dislocations, mainly those due to coronoid loss, are extremely difficult to treat and every effort has to be done in the acute setting in order to prevent their occurrence. It is important to keep in mind that even rather complex fracture-dislocations, unless open or associated with vascular or nervous injuries, are not a surgical emergency. It is safer to make an adequate surgical planning with a CT scan with bidimensional and tridimensional rendering that can help to better identify coronoid and radial head fractures, to prepare an adequate surgical setting with all the equipment for an adequate fixation, to have a radial head prosthesis and an external fixation system available, even if this means a delay of a few days.

In both simple and complex instabilities, when choosing between conservative and surgical treatment of the injured stabilizers, three cardinal rules should be followed at the same time.

- the elbow should be stably and concentrically reduced. A CT scan is sometimes necessary to assess the correct joint reduction, mainly in case of conservative treatment.

- the primary stabilizers should be repaired or, if treated conservatively, should be in such condition as to heal with a fast recovery of their mechanical properties.

- an early mobilization should be allowed, prolonged immobilization should be avoided, possibly never over 15-20 days.

As a consequence, in complex elbow instabilities the treatment is almost always surgical. In the recent years the concept that the quality of the functional result depends on the efficacy of the stabilizers repair and on how early the elbow mobilization is started has progressively gained credit [7].

Thus, coronoid fixation techniques and radial head fixation or prosthesis techniques [8] have improved. The importance has been recognized of repairing the LCL [9] and of having inside the surgical theater an external fixator available in order to keep the joint reduced when necessary. The role of pronation for ulnohumeral stabilization in case of LCL lesions [10] and of supination in case of MCL lesions [11] has been defined. Early active mobilization techniques, which avoid varus stress, have been introduced in the clinical practice in order to reduce the risk of elbow stiffness and to obtain the healing of some mild residual instability [12].

With respect to the above-mentioned rules, through an accurate diagnosis of the stabilizers lesions and their systematic and efficient repair, with the introduction of early rehabilitation protocols, it is certainly possible to obtain superior results if compared to the past [13].

It is however common experience for all those who treat elbow trauma to have a sense of frustration in front of some unsatisfactory results even after technically and methodologically correct treatments, especially for the advent of stiffness and heterotopic ossifications.

I think that challenges of the next future will be the prevention of heterotopic ossifications and the improvement of surgical instrumentations for radial head and coronoid internal fixation and for elbow external fixation. Defining a practical and shared classification will be in my opinion an important step forward for the comprehension and correct definition of the different and complex patterns of elbow instability and dislocation. 
Acknowledgments A special thank to my assistants Dr. Alessandro Marinelli, Dr. Enrico Guerra, Dr. Graziano Bettelli, Dr. Michele Cavaciocchi, with whom I assiduously discuss the complex traumatic elbow cases and who actively cooperated in the definition of the proposed classification.

Conflict of interest Roberto Rotini declares that he has no conflict of interest.

\section{References}

1. Celli A, Marongiu MC, Fontana M, Celli L (2008) The fracturedislocation of the forearm (Monteggia and Essex-Lopresti lesions) in Celli A, Celli L, Morrey BF (2008) Treatment of Elbow Lesions. Springer, Italia, Milan 2008:113-126

2. Giannicola G, Scacchi M, Sacchetti FM, Cinotti G (2013) Clinical usefulness of proximal ulnar and radial fracture-dislocation comprehensive classification system (PURCCS): prospective study of 39 cases. J Shoulder Elbow Surg 22(12):1729-1736

3. Morrey BF (1998) Complex instability of the elbow. AAOS Instr Course Lect 47:157-164

4. Hildebrand KA, Patterson SD, King GJ (1999) Acute elbow dislocations: simple and complex. Orthop Clin North Am 30(1):63-79

5. O'Driscoll SW, Morrey BF, Korinek S, An KN (1992) Elbow subluxation and dislocation. A spectrum of instability. Clin Orthop Relat Res 280:186-197

6. O'Driscoll SW, Jupiter JB, Cohen MS, Ring D, McKee MD (2003) Difficult elbow fractures: pearls and pitfalls. Instr Course Lect 52:113-134
7. Giannicola G, Sacchetti FM, Greco A, Cinotti G, Postacchini F (2010) Management of complex elbow instability. Musculoskelet Surg 94(Suppl 1):S25-S36

8. Rotini R, Marinelli A, Guerra E, Bettelli G, Cavaciocchi M (2012) Radial head replacement with unipolar and bipolar SBi system: a clinical and radiographic analysis after a 2-year mean follow-up. Musculoskelet Surg 96(Suppl 1):S69-S79

9. Johnson JA, Beingessner DM, Gordon KD, Dunning CE, Stacpoole RA, King GJ (2005) Kinematics and stability of the fractured and implant-reconstructed radial head. J Shoulder Elbow Surg 14(1):195S-201S

10. Dunning CE, Zarzour ZD, Patterson SD, Johnson JA, King GJ (2001) Ligamentous stabilizers against posterolateral rotatory instability of the elbow. J Bone Joint Surg Am 83A(12): $1823-1828$

11. Armstrong AD, Dunning CE, Faber KJ, Duck TR, Johnson JA, King GJ (2000) Rehabilitation of the medial collateral ligamentdeficient elbow: an in vitro biomechanical study. J Hand Surg Am 25(6): 1051-1057

12. Duckworth AD, Kulijdian A, McKee MD, Ring D (2008) Residual subluxation of the elbow after dislocation or fracturedislocation: treatment with active elbow exercises and avoidance of varus stress. J Shoulder Elbow Surg 17(2):276-280

13. Pugh DM, Wild LM, Schemitsch EH, King GJ, McKee MD (2004) Standard surgical protocol to treat elbow dislocations with radial head and coronoid fractures. $\mathrm{J}$ Bone Jt Surg Am $86 \mathrm{~A}(6): 1122-1130$ 